\title{
Microwave-Assisted Preparation of CdS Nanoparticles in a Halide-Free Ionic Liquid and Their Photocatalytic Activities
}

\author{
M. ESMAILI, A. HABIBI-YANGJEH* \\ Department of Chemistry, Faculty of Science, University of Mohaghegh Ardabili, P.O. Box 179, Ardabil, Iran
}

\begin{abstract}
A microwave-assisted (4-6 min) method was used for the preparation of CdS nanoparticles in 1-ethyl-3-methylimidazolium ethyl sulfate, a halide-free room-temperature ionic liquid (RTIL). The samples were characterized by powder X-ray diffraction, energy dispersive $\mathrm{X}$-ray spectroscopy, and scanning electron microscopy. Diffuse reflectance spectra showed a $1.33 \mathrm{eV}$ blue shift relative to bulk CdS. The photocatalytic activities of the nanoparticles for photodegradation of methylene blue (MB) using UV and visible light were measured. The photodegradation of MB decreased with calcination temperature. First order rate constants for the reaction under visible and UV irradiations over the nanoparticles prepared in the RTIL rich media were 5.4 and 2.5 higher, respectively, than the sample prepared in water.
\end{abstract}

Key words: nanoparticle; cadmium sulfide; room-temperature ionic liquid; photocatalysis; methylene blue

CLC number: O643 Document code: A

Received 11 January 2011. Accepted 16 February 2011.

*Corresponding author.Tel: +98-0451-5514702; Fax:+98-0451-5514701; E-mail: ahabibi@uma.ac.ir

This work was supported by University of Mohaghegh Ardabili.

English edition available online at Elsevier ScienceDirect (http://www.sciencedirect.com/science/journal/18722067).

Size, morphology, and dimensionality can strongly affect the properties of nanomaterials. Recently, nanostructured semiconductors with various structures and morphologies have received much attention due to their novel applications, intriguing properties, and quantum size effects [1,2]. One of the most important environmental applications of nanomaterials is their use as sensors with enhanced monitoring capabilities for pollutants. They are used for treating contaminated water, soil or air and in green technologies to eliminate or decrease harmful emissions and wastes from industry using photocatalytic processes [3-6]. Heterogeneous photocatalysis is a good method for the decontamination and mineralization of organic pollutants because of its high efficiency, low energy consumption and satisfactory environmental compatibility $[7,8]$.

As an important II-VI semiconductor $\left(E_{\mathrm{g}}=2.42 \mathrm{eV}\right), \mathrm{CdS}$ has promising applications in many technical fields including mechanical and optoelectronic fields, and use in solar cells, and the photodegradation of water pollutants [9-14]. The preparation of CdS nanoparticles has become a very popular research area in recent years [15-18].

Room temperature ionic liquids (RTILs) have been widely studied as a reaction media due to their unique physicochemical properties [19-21]. RTILs have recently received a great deal of attention as a new media for nanomaterial preparation [22-25]. One of the largest barriers to the application of RTILs in various fields is their high cost relative to conventional solvents. Moreover, the most commonly used RTILs have $\mathrm{PF}_{6}^{-}$and $\mathrm{BF}_{4}^{-}$ions [26]. RTILs with these ions are known to decompose in the presence of water and as a result, toxic and corrosive species are formed $[27,28]$. RTILs with alkyl sulfate anions are halogen-free and relatively hydrolysis-stable and they could be a better alternative for industrial applications [29].

Microwave irradiation application to material synthesis has shown very rapid growth due to its unique reaction effects such as rapid volumetric heating and the consequent dramatic increase in reaction rates, etc. [30,31]. RTILs are excellent microwave-absorbing agents due to their high ionic conductivity and polarizability, thus leading to a very high heating rate and a significantly shortened reaction time [32]. Recently, various nanomaterials such as $\mathrm{M}_{2} \mathrm{~S}_{3}(\mathrm{M}=\mathrm{Bi}$, $\mathrm{Sb}), \mathrm{ZnO}, \mathrm{CuO}$, and $\mathrm{SnO}_{2}$ have been prepared in RTILs using microwave irradiation [33-35]. In the preparation of nanomaterials with microwave irradiations, it has been mainly RTILs with $\mathrm{PF}_{6}^{-}$and $\mathrm{BF}_{4}^{-}$ions that have been used [35-37]. Moreover, the effect of the RTIL on the photocatalytic activity of the prepared nanomaterials has been rarely examined [38].

In this paper, a fast, template-free and green method was applied for the preparation of $\mathrm{CdS}$ nanoparticles in 1-ethyl-3-methylimidazolium ethyl sulfate ([EMIM] [EtSO $\left.\left.{ }_{4}\right]\right)$ as a halide-free and low cost RTIL [39]. This can give a cost-effective, fast, and simple method for the prepa- 
ration of CdS nanoparticles with improved photocatalytic activity on a large scale.

\section{Experimental}

\subsection{Materials}

Cadmium acetate $\left(\mathrm{Cd}\left(\mathrm{CH}_{3} \mathrm{COO}\right)_{2} \cdot 2 \mathrm{H}_{2} \mathrm{O}\right.$, extra pure), thioacetamide (TAA, $\mathrm{CH}_{3} \mathrm{CSNH}_{2}$, GR for analysis) and absolute ethanol were obtained from Merck and used without further purification. The ionic liquid was synthesized according to the literature [39]. Double distilled water was used for the experiments.

\subsection{Instruments}

The X-ray diffraction (XRD) patterns were recorded on a Philips Xpert X-ray diffractometer with $\mathrm{Cu} K_{\alpha}$ radiation $(\lambda=$ $0.15406 \mathrm{~nm}$ ) employing a scan rate of $1 \%$ min in the $2 \theta$ range from $20^{\circ}$ to $80^{\circ}$. Surface morphology and the distribution of particles were characterized by a LEO 1430VP scanning electron microscopy (SEM) using an accelerating voltage of $15 \mathrm{kV}$. The purity and elemental analysis of the products were obtained by energy dispersive analysis of X-rays (EDX) on the same LEO 1430VP instrument with the accelerating voltage of $20 \mathrm{kV}$. The samples used for SEM and EDX observations were prepared by transferring the particles, which were first dispersed in ethanol, to a glass substrate attached to the SEM stage. After the evaporation of ethanol from the substrate, the particles on the stage were coated with a thin layer of gold and palladium. Diffuse reflectance spectra (DRS) were recorded by a Scinco 4100 apparatus.

\subsection{Preparation of CdS nanoparticles}

In a typical synthesis procedure, cadmium acetate dihydrate $(5.06 \mathrm{~g})$ was dissolved in $12.5 \mathrm{ml}$ of distilled water and $12.5 \mathrm{ml}$ of the RTIL under stirring at room temperature. Also, $1.50 \mathrm{~g}$ of TAA was dissolved in $12.5 \mathrm{ml}$ of distilled water and $12.5 \mathrm{ml}$ of the RTIL. Then the TAA solution was slowly added into the solution of cadmium acetate under magnetic stirring. The solution was irradiated for $4 \mathrm{~min}$ with $55 \%$ output using a domestic microwave oven. The yellow color suspension formed was centrifuged to recover the precipitate and washed three times with double distilled water and ethanol, respectively, to remove unreacted reagents and was dried in an oven at $50{ }^{\circ} \mathrm{C}$ for $24 \mathrm{~h}$. To investigate the effect of the RTIL on the properties of the product, one more comparative sample was prepared by keeping the reaction parameters constant except that the product was prepared in water as solvent. In order to demonstrate the effect of solvent composition, the CdS nanoparticles were prepared in aqueous solutions of the RTIL with 1:4 composition ( $10 \mathrm{ml}$ water $+40 \mathrm{ml}$ RTIL). Also, to demonstrate the effect of microwave irradiation time with the RTIL rich media, one more comparative sample was prepared using 6 min microwave irradiation.

\subsection{Photocatalysis experiments}

To examine the photocatalytic activity, the photodegradation of methylene blue (MB), which is a typical dye resistant to biodegradation, was investigated. A photochemical reactor with a water circulation arrangement to maintain the temperature at $25{ }^{\circ} \mathrm{C}$ was used. The solution was magnetically stirred and continuously aerated by a pump to provide oxygen and complete mixing of the reaction solution. A UV Osram lamp of $125 \mathrm{~W}$ with the major fraction of irradiation occurring at $365 \mathrm{~nm}$ and a tungsten lamp of $150 \mathrm{~W}$ as visible source were used. The lamps were fitted on the top of the reactor. Prior to illumination, a suspension containing $0.1 \mathrm{~g}$ of the nanoparticle and $250 \mathrm{ml}$ of $\mathrm{MB}\left(3 \times 10^{-5} \mathrm{~mol} / \mathrm{L}\right)$ was stirred continuously in the dark for $30 \mathrm{~min}$ to attain adsorption equilibrium. Samples were taken from the reactor at regular intervals and centrifuged to remove the photocatalyst before analysis by the spectrophotometer at $664 \mathrm{~nm}$, which is the maximum absorption wavelength $\left(\lambda_{\max }\right)$ of MB. The adsorption capacities, $q_{\mathrm{e}}(\mathrm{mol} / \mathrm{g})$, of the nanoparticles were calculated by a mass balance relationship that represented the amount of adsorbed dye per amount of the photocatalyst:

$$
q_{\mathrm{e}}=\frac{\left(c_{0}-c_{\mathrm{e}}\right) V}{W}
$$

where $c_{0}$ and $c_{\mathrm{e}}$ were concentrations of the dyes $\left(\mathrm{mol} / \mathrm{dm}^{3}\right)$ at $t=0$ and time of equilibrium. $V$ was the volume of the solution $\left(\mathrm{dm}^{3}\right)$, and $W$ was the weight of dry adsorbent $(\mathrm{g})$. In order to prevent photocatalytic degradation of the dye, the adsorption experiments were carried out in the dark.

\section{Results and discussion}

To investigate the effect of the solvent on the properties of the CdS nanoparticles, comparative experiments were carried out. The phase and purity of the samples were determined by XRD. Typical diffraction patterns are shown in Fig. 1. The diffraction peaks corresponded to the (111), (200), (220), (311), and (331) planes of the cubic CdS crystal system (JCPDS 42-1411). No peaks from possible impurities were observed. It is clear that all the products have the same cubic crystal structure and the peak broadening in the patterns indicated that the nanoparticles were very small in size. The XRD data were also used to estimate the average 


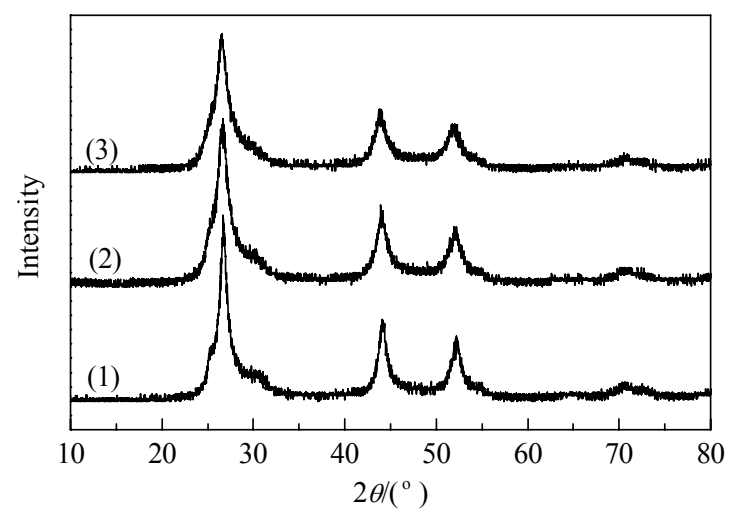

Fig. 1. The powder XRD patterns for the CdS nanoparticles prepared using 4 min microwave irradiation in water (1), aqueous solution of the RTIL with 1:1 (2), and 1:4 compositions (3).

size of the crystallites by the Scherrer's equation [40]:

$$
D=K \lambda / B \cos \theta
$$

where $D$ was average crystallite sizes, $\lambda$ the wavelength of $\mathrm{X}$-ray radiation $(0.15406 \mathrm{~nm}), K$ the Scherrer's constant $(K$ $=0.9), \theta$ the characteristic X-ray radiation $\left(2 \theta=44.30^{\circ}\right)$ and $B$ was the full-width-at-half-maximum of the (220) plane (in radians). The mean crystallite sizes of the nanoparticles prepared in water, aqueous solutions of the RTIL with 1:1 (1 water + 1 RTIL, in volume) and 1:4 compositions (1 water + 4 RTIL, in volume) with 4 min microwave irradiation were 8,6 , and $4 \mathrm{~nm}$, respectively. The mean crystallite size of the nanoparticles decreased as the amount of the RTIL increased in the reaction media.

The morphology of the nanoparticles prepared in aqueous solution of the RTIL with 1:1 and 1:4 compositions using 4 min microwave irradiation was investigated by SEM, which are shown at different magnifications in Fig. 2. It is evident that the structure of the nanoparticles was nanospheres with different sizes. It is clear that aggregation of the nanoparticles decreased with increasing RTIL content in the media.

The purity and composition of the product were studied by energy dispersive X-ray spectroscopy (EDX). The results are displayed in Fig. 3. The curves showed Cd and S peaks

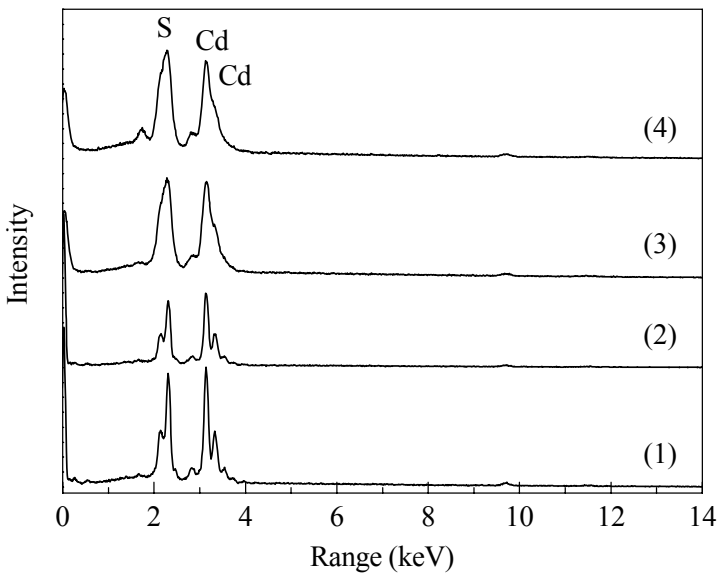

Fig. 3. EDX patterns of CdS nanoparticles prepared with $4 \mathrm{~min}$ microwave irradiation in water (1), aqueous solution of the RTIL with 1:1 (2) and 1:4 compositions with 4 min irradiation (3), and 1:4 composition with $6 \mathrm{~min}$ irradiation (4).

and the average atomic percentage ratio of $\mathrm{Cd}: \mathrm{S}$ for nanoparticles prepared in the aqueous solution of the RTIL with 1:1 composition (using $4 \mathrm{~min}$ irradiation) and the aqueous solution of the RTIL with 1:4 compositions using 4 and $6 \mathrm{~min}$ irradiations were 55.3:44.7, 55.6:44.4, and 53.1:46.9, respectively. The other peaks in the figure corresponded to gold, palladium, and silicate which were due to sputter coating of the glass substrate on the EDX stage, and these were not considered in the elemental analysis of $\mathrm{Cd}$ and S. It is clear that the CdS nanoparticles prepared were sufficiently pure.

Figure 4 shows the DRS of the nanoparticles. The absorption maximum for the CdS nanoparticles was $330 \mathrm{~nm}$. The band gap energy for the nanoparticles $(3.75 \mathrm{eV})$ was increased compared to that of bulk CdS $(2.42 \mathrm{eV})$ [41]; the increase in the band gap $(1.33 \mathrm{eV})$ or blue shift can be attributed to the quantum confinement effect of the nanoparticles. As can be seen, the nanoparticles have good absorption in UV and the visible range of spectrum. Therefore, the nanoparticles should have photocatalytic activity in the UV
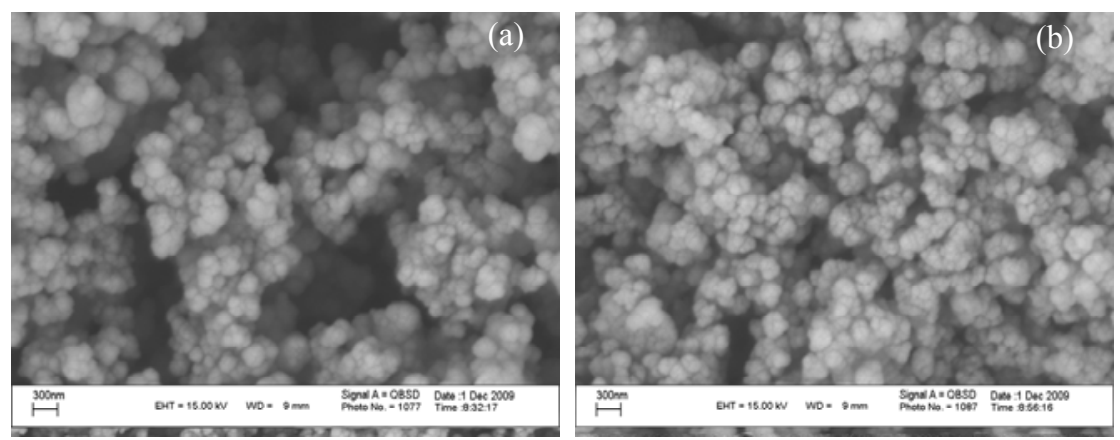

Fig. 2. SEM images of CdS nanoparticles prepared in aqueous solution of the RTIL with 1:1 (a) and 1:4 (b) compositions using 4 min microwave irradiation. 


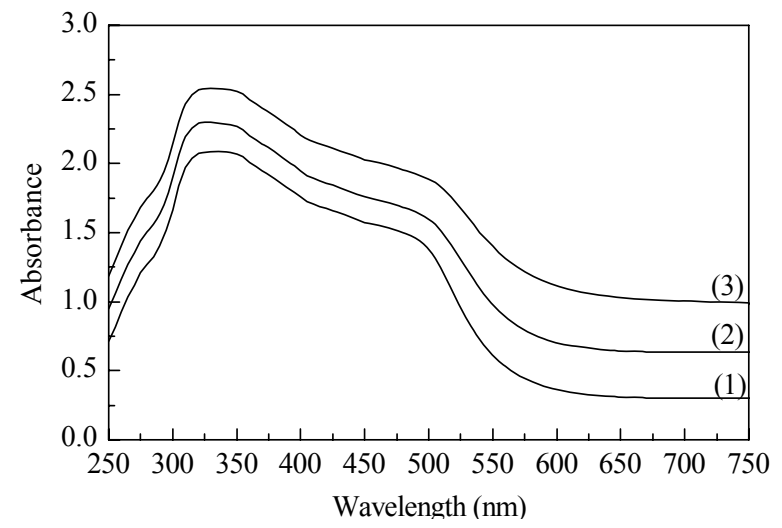

Fig. 4. DRS of CdS nanoparticles prepared using 4 min microwave irradiation in water (1), aqueous solution of the RTIL with 1:1 (2) and 1:4 compositions (3).

and visible regions.

To investigate the photocatalytic activity of the samples, the photodegradation of MB was studied. In Fig. 5, the results from the photodegradation of MB by UV irradiation on the nanoparticles prepared in aqueous solution of the RTIL with 1:1 composition calcined at various temperatures are shown. Generally, the photocatalytic activity depends on calcination temperature [42]. Figure 5 demonstrates that the photodegradation of $\mathrm{MB}$ was decreased with calcination temperature. Also, similar results were obtained for the nanoparticles prepared in aqueous solution of the RTIL with 1:4 composition using 4 and 6 min microwave irradiations (Supplement 1). The decrease in the photocatalytic activity with calcination temperature may be due to aggregation of the nanoparticles at higher temperatures. In order to compare the photocatalytic activity of the nanoparticles prepared in the various media, the photodegradation of $\mathrm{MB}$ on the nanoparticles under UV irradiation is given in Fig. 6. The

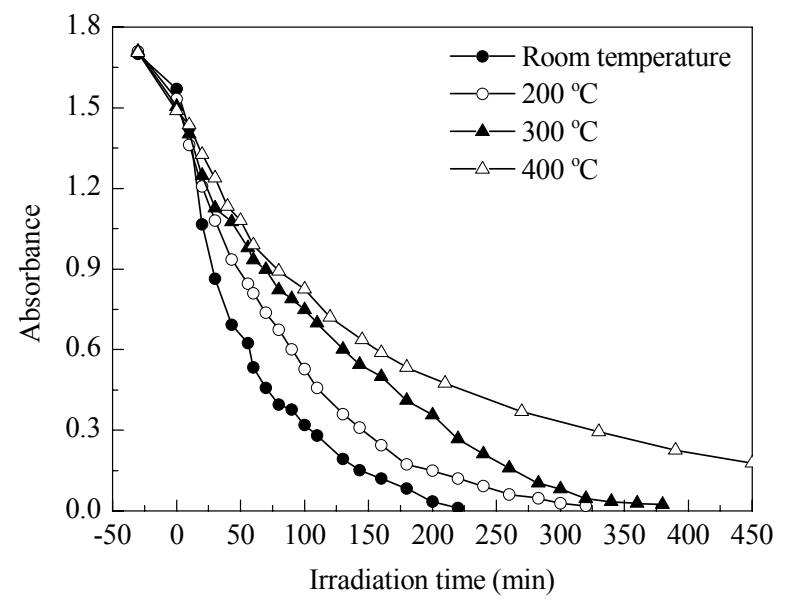

Fig. 5. Photodegradation of $\mathrm{MB}$ by UV irradiation on the CdS nanoparticles prepared in an aqueous solution of the RTIL with 1:1 composition using 4 min microwave irradiation calcined at various temperatures. photodegradation of $\mathrm{MB}$ on $\mathrm{CdS}$ nanoparticles (without calcination) prepared in aqueous solutions of the RTIL with 1:4 composition was higher than the samples prepared under other conditions. The complete degradation of $\mathrm{MB}$ on the nanoparticles prepared in the RTIL rich media occurred at $180 \mathrm{~min}$, which was very much less than the corresponding time for the sample prepared in water.

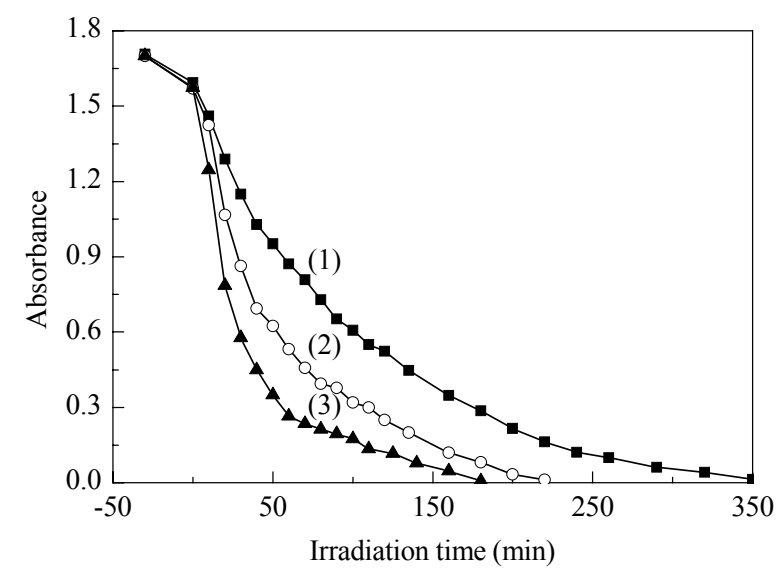

Fig. 6. Photodegradation of MB by UV irradiation on the CdS nanoparticles prepared in water (1), aqueous solution of the RTIL with 1:1 (2), and 1:4 (3) compositions using 4 min microwave irradiation.

To compare the photocatalytic activity of the nanoparticles under visible light irradiation, the photodegradation of MB was examined and the results are shown in supplement 2. Similar to the application of UV irradiation, the photodegradation of $\mathrm{MB}$ on the $\mathrm{CdS}$ nanoparticles under visible light irradiation decreased with calcination temperature. To compare the photocatalytic activity of the nanoparticles prepared at various conditions, the results of the photodegradation of MB under visible light on the samples prepared without calcination are given in Fig. 7. Similar to UV irradiation, the photodegradation of MB under visible light on $\mathrm{CdS}$ nanoparticles prepared in aqueous solutions of the RTIL with 1:4 composition was higher than the samples prepared under other conditions. The complete degradation of $\mathrm{MB}$ on the CdS nanoparticles prepared in the RTIL rich media occurred at $550 \mathrm{~min}$, which was very much less than the corresponding time for the other samples. Thus, the photocatalytic activities of the nanoparticles prepared in the RTIL rich media under UV and visible light irradiations were considerably higher than the sample prepared with water.

The observed first-order rate constant $\left(k_{\mathrm{obs}}\right)$ for the photocatalytic degradation of MB over the CdS nanoparticles were calculated using equation (3) [43]:

$$
\ln \frac{[\mathrm{MB}]}{[\mathrm{MB}]_{0}}=-k_{\mathrm{obs}} t
$$

in which $[\mathrm{MB}]$ and $[\mathrm{MB}]_{0}$ were the concentrations of $\mathrm{MB}$ 


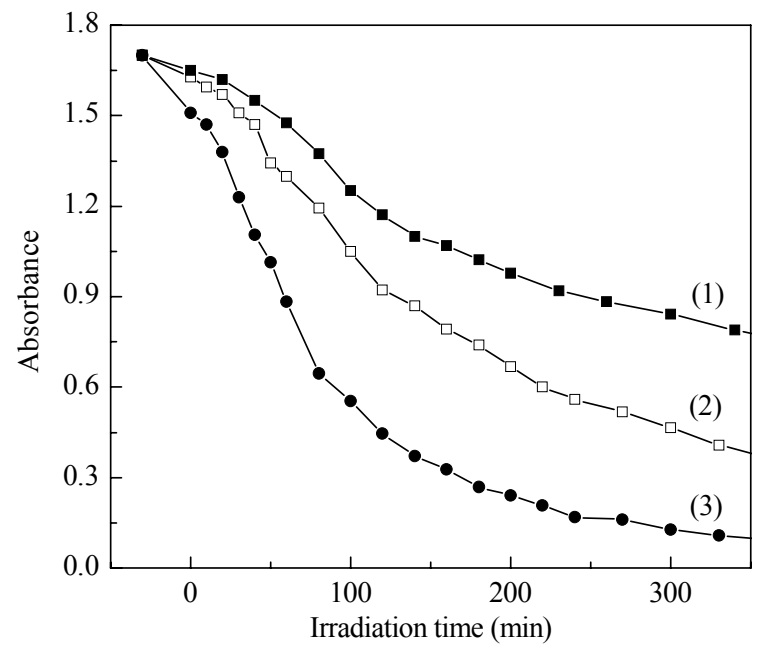

Fig. 7. Photodegradation of MB by visible light on CdS nanoparticles prepared in water (1), aqueous solution of the RTIL with 1:1 (2), and 1:4 compositions (3) using 4 min microwave irradiation.

(mol/L) at time $t$ and $t=0$ ( $t$ was the irradiation time). The first-order rate constants for the photocatalytic degradation of $\mathrm{MB}$ over the CdS nanoparticles prepared under various conditions were calculated using plots of $\ln [\mathrm{MB}]$ or $\ln A$ (logarithm of absorbance) versus irradiation time. The results are tabulated in Table 1 . The rate constant of the reaction increased with the RTIL content in the media and microwave irradiation time. The first order rate constant of the reaction with the nanoparticles prepared in the RTIL rich media at 6 min microwave irradiation under visible and UV lights were 5.4 and 2.5 times more, respectively, than the sample prepared in water.

The photocatalytic activity of semiconductors is mainly determined by crystal structure, surface area, size of particles, band gap energy, and morphology [44]. The small-sized nanoparticles with high surface areas were effective substrates for the adsorption of pollutant molecules and UV and visible lights [38]. To compare ability of the nanoparticles for the adsorption of MB molecules, the adsorption capacity $\left(q_{\mathrm{e}}\right)$ for the nanoparticles was calculated using equation (1) after 90 min adsorption in the dark [45]. The values of $q_{\mathrm{e}}$ for $\mathrm{MB}$ adsorption on the nanoparticles prepared in water, aqueous solution of the RTIL with 1:1 composition, aqueous solution of the RTIL with 1:4 composition using 4 and 6 min microwave irradiations were $8.32 \times$ $10^{-6}, 9.50 \times 10^{-6}, 1.09 \times 10^{-5}$, and $1.45 \times 10^{-5} \mathrm{~mol} / \mathrm{g}$, respectively. It is clear that the adsorption capacity for MB molecules on the nanoparticles prepared with the RTIL was higher than that of the sample prepared in water. Therefore, significant improvement in the photocatalytic activity for the nanoparticles prepared in presence of the RTIL could be due to the higher surface area of the nanoparticles due to less aggregation. Moreover, a limiting factor that controls the efficiency of photocatalysis is the rapid recombination of photogenerated electrons and holes in the semiconductor particles [46]. Recombination of the electron-hole pair within semiconductor particles is drastically reduced with decreasing particle size [47]. Hence, due to the smaller size, the recombination rate of electron and hole for the nanoparticles prepared with the RTIL would be lower than that of the sample prepared in water. For these reasons, the photocatalytic activity of the CdS nanoparticles prepared in the RTIL rich media was higher than that of the sample prepared in water.

Table 1. First-order rate constants for the photocatalytic degradation of $\mathrm{MB}$ on the $\mathrm{CdS}$ nanoparticles prepared in water, aqueous solutions of the RTIL with 1:1 and 1:4 compositions using 4 and 6 min irradiations

\begin{tabular}{lcccc}
\hline \multirow{4}{*}{ Light } & \multicolumn{4}{c}{$k_{\text {obs }} / \mathrm{min}^{-1}$} \\
\cline { 2 - 5 } & Water & $\begin{array}{c}25 \text { Water } \\
+25 \mathrm{RTIL}\end{array}$ & $\begin{array}{c}10 \text { Water } \\
+40 \mathrm{RTIL}^{\mathrm{a}}\end{array}$ & $\begin{array}{c}10 \text { Water } \\
+40 \mathrm{RTIL}^{\mathrm{b}}\end{array}$ \\
\hline Visible & $22.9 \times 10^{-4}$ & $40.3 \times 10^{-4}$ & $74.9 \times 10^{-4}$ & $123 \times 10^{-4}$ \\
UV & $11.0 \times 10^{-3}$ & $15.9 \times 10^{-3}$ & $20.0 \times 10^{-3}$ & $27.4 \times 10^{-3}$ \\
\hline
\end{tabular}

${ }^{a}$ Microwave irradiation time was $4 \mathrm{~min}$.

${ }^{\mathrm{b}}$ Microwave irradiation time was $6 \mathrm{~min}$.

The RTIL, [EMIM][EtSO ${ }_{4}$, consists of $\left[\mathrm{EMIM}^{+}\right.$cations and $\left[\mathrm{EtSO}_{4}\right]^{-}$anions. When an aqueous solution of the RTIL is used as a reaction medium, the sulfide moieties of the $\mathrm{CdS}$ nuclei was highly solvated by $[\mathrm{EMIM}]^{+}$ions by electrostatic and hydrogen bonding interactions $[38,48,49]$. Hydrogen bonding interactions formed between the hydrogen atoms at position 2 of the imidazolium ring and the sulfide ions of the CdS crystal cores and the electrostatic interaction between them may act as an effective bridge to connect the nuclei of cadmium sulfide and cations of the RTIL. This would play a crucial role in the inhibition of nanoparticle growth and aggregation. Moreover, the low interfacial tension of RTILs gave high nucleation rates, thus enabling the generation of small nanoparticles [50]. For these reasons, similar to our previous works [51,52], the size and aggregation of the CdS nanoparticles prepared in the presence of the RTIL were lower than that of the sample prepared in water. Hence, the nanoparticles prepared in presence of the RTIL would have higher photocatalytic activity.

\section{Conclusions}

Microwave irradiation for 4-6 min was applied for the preparation of $\mathrm{CdS}$ nanoparticles in [EMIM] $\left[\mathrm{EtSO}_{4}\right]$ as a low cost and halide-free RTIL. This environmentally benign method is fast and template-free, and remarkably shortened preparation time and avoided complicated preparation procedures. The photocatalytic activities of the nanoparticles 
prepared in an aqueous solution of the RTIL under UV and visible lights were considerably higher than those of the sample prepared in water. The photocatalytic activity decreased with the calcination temperature of the nanoparticles.

\section{Acknowledgement}

The authors wish to acknowledge University of Mohaghegh Ardabili for financial support of this work.

\section{References}

1 Cui Y, Lieber C M. Science, 2001, 291: 851

2 Hu H, Zhang W. Opt Mater, 2006, 28: 536

3 Reddy M P, Venugopal A, Subrahmanyam M. Appl Catal B, 2006, 69: 164

4 Wu C H, Chern J M. Ind Eng Chem Res, 2006, 45: 6450

5 Dalrymple O K, Yeh D H, Trotz M A. J Chem Technol Biotechnol, 2007, 82: 121

6 Teichner S J. J Porous Mater, 2008, 15: 311

7 Su W, Chen J, Wu L, Wang X, Wang X, Fu X. Appl Catal B, 2008, 77: 264

8 Mohamed S H. J Phys D, 2010, 43: 35406

9 Fuhrer M S, Nygard J, Shih L, Forero M, Yoon Y G, Mazzoni M S C, Choi H J, Louie S G, Zettl A, Metuen P L. Science, 2000, 288: 494

10 Li H L, Zhu Y C, Chen S G, Palchik O, Xiong J P, Koltypin Y, Gofer Y, Gedanken A. J Solid State Chem, 2003, 172: 102

11 Datta A, Priyam A, Bhattacharyya S N, Mukherjea K K, Saha A. J Colloid Interface Sci, 2008, 322: 128

12 Zong X, Yan H, Wu G, Ma G, Wen F, Wang L, Li C. J Am Chem Soc, 2008, 130: 7176

13 Yu X X, Yu J G, Cheng B, Huang B B. Chem Eur J, 2009, 15: 6731

14 Yu J, Zhang J, Jaroniec M. Green Chem, 2010, 12: 1611

15 Lu X, Mao H, Zhang W, Wang C. Mater Lett, 2007, 31: 2288

16 Shen S, Gao L. Mater Res Bull, 2008, 43: 437

17 Barzegar M, Habibi-Yangjeh A, Behboudnia M. J Phys Chem Solids, 2010, 71: 1393

18 Taghvaei V, Habibi-Yangjeh A, Behboudnia M. J Iran Chem Soc, 2010, 7: S175

19 Zhang Z C. Adv Catal, 2006, 49: 153

20 Jacob D S, Bitton L, Grinblat J, Felner I, Koltypin Y, Gedanken A. Chem Mater, 2006, 18: 3162

21 Parvulescu V I, Hardacre C. Chem Rev, 2007, 107: 2615

22 Wang Y, Yang H. J Am Chem Soc, 2005, 127: 5316

23 Farag H K, Endres F. J Mater Chem, 2008, 18: 442

24 Cao S W, Zhu Y J, Cheng G F, Huang Y H. J Hazard Mater
2009, 171: 431

25 Xu C, Luo H, Liu W, Ying T. Ceramics Intern, 2009, 35: 917

26 Keskin S, Kayrak-Talay D, Akman U, Hortacsu O. J Supercritical Fluids, 2007, 43: 150

27 Najdanovic-Visak V, Esperanca J M S S, Rebelo L P N, da Ponte M N, Guedes H J R, Seddon K R, Szydlowski J. Phys Chem Chem Phys, 2002, 4: 1701

28 Cammarta L, Kazarian S G, Salter P A, Welton T. Phys Chem Chem Phys, 2001, 23: 5192

29 Wasserscheid P, Hal R, Bosmann A. Green Chem, 2002, 4: 400

30 Panda A B, Glaspell G, El-Shall M S. J Am Chem Soc, 2006, 128: 2790

31 Xiang Q J, Yu J G, Cheng B, Ong H C. Chem Asian J, 2010, 5: 1466

32 Cao S W, Zhu Y J. Acta Materialia, 2009, 57: 2154

33 Jiang Y, Zhu Y J. J Phys Chem B, 2005, 109: 4361

34 Wang W W, Zhu Y J, Cheng G F, Huang Y H. Mater Lett, 2006, 60: 609

35 Liu H, Liang Y, Hu H, Wang M. Solid State Sci, 2009, 11: 1655

36 Dong W S, Li M Y, Liu C, Lin F, Liu Z. J Colloid Interface Sci, 2008, 319: 115

37 Xu X, Zhang M, Feng J, Zhang M. Mater Lett, 2008, 62: 2787

38 Wang L, Chang L, Zhao B, Yuan Z, Shao G, Zheng W. Inorg Chem, 2008, 47: 1443

39 Gomez E, Gonzalez B, Calvar N, Tojo E, Dominguez A. J Chem Eng Data, 2006, 51: 2096

40 Cullity B D. Elements of X-Ray Diffraction, 2nd Ed. London: Addision Wesley, 1978

41 Arora S, Manoharan S S. Mater Chem Phys, 2008, 110: 34

42 Baiju K V, Shukla S, Sandhya K S, James J, Warrier K G K. J Phys Chem C, 2007, 111: 7612

43 Behnajady M A, Modirshahla N, Hamzavi R. J Hazard Mater B, 2006, 133: 226

44 Testino A, Bellobono I R, Buscagalia V, Canevali C, Darienzo M, Plolizzi S, Scotti R, Morazzoni F. J Am Chem Soc, 2007, 129: 3564

45 Wang S, Li H, Xu L. J Colloid Interface Sci, 2006, 295: 71

46 Robert D. Catal Today, 2007, 122: 20

47 Stroyuk A L, Kryukov A I, Ya Kuchmii S, Pokhodenko V D. Theor Exper Chem, 2005, 41: 207

48 Harifi Mood A R, Habibi-Yangjeh A, Gholami M R. J Phys Chem B, 2006, 110: 7073

49 Biswas K, Rao C N R. Chem Eur J, 2007, 13: 6123

50 Antonietti M, Kuang D, Smarsly B, Yong Z. Angew Chem Int Ed, 2004, 43: 4988

51 Behboudnia M, Habibi-Yangjeh A, Jafari-Tarzanag Y, Khodayari A. J Crys Growth, 2008, 310: 4544

52 Taghvaei V, Habibi-Yangjeh A, Behboudnia M. Powder Technol, 2009, 195: 63 